\title{
The Role of Learner Characteristics in the Adaptive Educational Hypermedia Systems: The Case of the MATHEMA
}

\author{
Alexandros Papadimitriou \\ Department of Electrical and Electronic Engineering, School of Technological and Pedagogical Education of Athens, \\ Athens, 15122, Greece \\ Email: apapadim@di.uoa.gr \\ Georgios Gyftodimos \\ Department of Philosophy and History of Science, University of Athens, Athens, 10558, Greece \\ Email: gyftodim@phs.uoa.gr
}

Received: 16 June 2017; Accepted: 25 July 2017; Published: 08 October 2017

\begin{abstract}
The aim of this paper is to explore the characteristics of the learners used by the developed adaptive educational hypermedia systems to date to draw conclusions about their relation to the adaptation techniques they use and to be explained the rationale for selecting of the learners' characteristics used by the adaptive educational hypermedia system MATHEMA for its adaptation techniques. At first, the characteristics of learners using some systems for their adaptive techniques are presented. Here is the presentation of learner's characteristics used by adaptation techniques of the adaptive educational hypermedia system MATHEMA. Finally, an evaluation of the main functions of the MATHEMA is performed. In conclusion, we discuss the choice of specific learners' characteristics for adaptation techniques of the MATHEMA and the resulting educational benefits.
\end{abstract}

Index Terms-Adaptive educational hypermedia systems, learner characteristics, adaptation techniques

\section{INTRODUCTION}

The rapid development of the Internet and the Web over recent years has led to an increasing interest in creating Web-based learning tools and learning environments, such as the Adaptive Educational Hypermedia Systems (AEHSs).

Hypermedia consists of different media and integration, such as text, graphic, animation, audio, etc. not only have various media and their integration greatly enriched the learning environment, but also the production of multimedia teaching material. Learners and hypermedia systems can freely realize man-machine interaction [25].

The term adaptation in e-Learning systems involves the selection and manner of presentation of each learning activity as a function that examines the entity of knowledge, skills and other information given by each subject taught [71].

AEHSs combine ideas from hypermedia and intelligent tutoring systems (ITS) in order to produce applications whose the content, link structures, and other features are dynamically adapted to the learner's characteristics, such as the learning goal, current task, knowledge level, performance, background-experience, interests, preferences, stereotypes, cognitive preferences, learning or cognitive style, personal data, abilities/disabilities, social group, working environment, demographic data, and situation variables. All these characteristics stored in the learner's model, play an important role both in the development and the functionality of the AEHSs.

According to [11], AEHSs could be considered as the solution to the problems of "traditional" online educational hypermedia systems, due mainly to their static content, the "lost in hypermedia" syndrome and the "one-size-fits-all" approach. Furthermore, AEHSs, increase the functionality of the conventional hypermedia by combining free browsing with personalization and can support all the continuum of the learning model, from the pure system-controlled to the fully learner-controlled.

In the Web-based AEHSs, several adaptive and intelligent techniques have been applied to introduce adaptation, such as [11]:

(a) Curriculum Sequencing: It helps the learner to follow an optimal path through the learning material.

(b) Adaptive Presentation: It adapts the content presented in each hypermedia node according to specific characteristics of the learner.

(c) Adaptive Navigation Support: It adapts the link structure in such a way to guide the learner towards interesting and relevant information, kept away from non-relevant information either by suggesting the most 
relevant links follow or by providing adaptive comments with visible links.

(d) Meta-adaptive Navigation Support: It selects or suggests the most appropriate adaptive navigation technique that suits the given learner best relative to the given context, either by observing and evaluating the success of each technique in different contexts and the resulting learning from these observations, or by assisting the learner in selecting the navigation technique that best suits to him/her.

(e) Interactive Problem-Solving Support: It provides the learner with intelligent help on every step of problem-solving, by giving a hint to executing the next step for the learner.

(f) Intelligent Analysis of Learner's Solutions: It uses intelligent analysers that not only tell the learner, whether the solution is correct, but also it tells him or her what exactly is wrong or incomplete.

(g) Example-based Problem-Solving Support: It helps the learners in solving new problems, not by articulating their errors, but by suggesting them relevant successful problem-solving cases, chosen from their earlier experience.

(h) Adaptive Collaboration Support; Adaptive Group Formation and/or Peer Help: These techniques support the collaboration process either just like the interactive problem-solving support systems help an individual learner in solving a problem, or they use knowledge about possible collaborating peers to form a matching group relative to the kind of the collaborative task.

According to [11], the adaptive navigation support is now a popular research area while the adaptive presentation is the most popular and the most studied method of hypermedia adaptation.

Two significant terms using in the AEHSs are the adaptability and adaptivity. According to [6], the adaptability refers to the capacity of adaptive learning systems to automatically adapt the learning process to the specific requirements and preferences of a particular learner. According to [62], the adaptivity could be defined as the capability of an adaptive learning system to alter its behaviour according to learner needs and other characteristics.

First generation systems provided limited adaptability through stereotype-based user models and limited functionality adaptation techniques, such as direct guidance, stretch text, hiding, and primitive link annotation. An example of such a system is the ISISTutor. Second generation systems have improved upon the functionality of first generation systems, introduced new capabilities, such as adaptive multimedia presentation, map adaptation, and link sorting. Examples of such systems are the ELM-ART, AHA!, and CS383. Third generation adaptive hypermedia systems support multidimensional user models that improve their functionality and enhance the support offered to learners. INSPIRE and TANGOW are examples of such systems.

In this paper, the aim is to investigate the AEHSs developed so far in order to find out which of the learner characteristics used by them for various kinds of adaptation to compare with the characteristics that the MATHEMA uses for various kinds of its adaptation support so that to draw conclusions about the relationship of student characteristics with adaptive techniques (rationale for selecting students' characteristics for adaptation).

The rest of the paper is organized as follows: In Section II, we present related works on the learner characteristics used for adaptation in AEHSs. In Section III, we refer to the MATHEMA and the learner characteristics that it uses in its adaptation techniques. In Section IV, we present the evaluation of the MATHEMA about its functions. In Section V, we summarize the most significant points of our work and we refer to our future plans.

\section{RELATED WORKS}

The bibliographic method used in this research is the state-of-the-art review about AEHSs and specifically for the learner's characteristics used for their adaptation techniques. For this aim, we made an extended research to draw conclusions about the selection of a concrete learner's characteristic for a concrete adaptation technique.

Educators know that individual learner characteristics play a huge role in how fast and in how well overall learning occurs. According to [11], an AEHS is based upon the assumption that each learner has different characteristics and that different educational settings could be more suitable for one type of learner than for another. Whenever, course content could be provided in a flexible way, adapted to individual learners' characteristics through the e-learning system, the system can deliver the course content so that it capitalizes on the learner's characteristics to optimize the learning outcome.

According to [2], an adaptive e-learning system based on the learner knowledge and learning style has a higher level of perceived usability than a non-adaptive elearning system. As usability influencing on the learner's satisfaction, engagement, and motivation when using elearning systems, learning enhancement expected when the system is highly usable.

According to [69], the learning process in an actual AEHS environment is complex and influenced by many characteristics of the learner. It is therefore important to consider accommodating as many of these characteristics as possible into the learner model to generate an exact adaptation. However, many learner characteristics have been identified in the literature; it is, therefore, important to select for use in the learner model only those characteristics that directly influence learner achievement in the specific learning process, otherwise, the design of the learner model will become unnecessarily complex. Thus, the [69] suggest that the learner characteristics being considered in an AEHS are knowledge level, learning styles, experience, background, and preference. 
Other characteristics, such as age, sex/gender, race/ethnicity, demographic data, interests, etc. are not taken into account as they are considered much less influencing the learner's achievement.

According to [24], an important issue in AEHSs is to investigate the characteristics of the learner, to specify on which ones the educational process should be adapted. The goals of the learner, but also their background [11], knowledge level [28], experience and learning style [69] perceived as characteristics that differentiate the users of a system and considered very important on the influence they may have on the learning process. According to [73], a profile could be considered complete when it incorporates the users' perceptual preference characteristics that mostly deal with the intrinsic parameters.

A research of [49] showed that learning systems' adaptation is highly successful when one or more of the following learner characteristics adapted: learning styles, cognitive styles, background knowledge, preferences (for particular types of learning materials), and motivation.

Also, research of [3] indicated that the adaptation based on the combination of the information perception, learning style, and knowledge level yields a much better learning outcome (both in the short- and long-term) and learner satisfaction than adaptation based on either of these learner characteristics alone; this combination is also marked by a much higher level of perceived usability compared to a non-adaptive version of the elearning system.

A research of [22] on learners with differing knowledge and motivation indicated that low prior knowledge learners benefited more than high prior knowledge learners. A research of [60] indicated that the learners with more favourable characteristics (i.e., higher prior knowledge, more complex epistemological beliefs, more positive attitudes towards mathematics, better cognitive and meta-cognitive strategy use) tended to show a more adaptive example utilization behaviour, reported less cognitive load, and solved more problems correctly than learners with less favourable characteristics.

According to [17], many studies have found that learners with different levels of prior knowledge benefit differently in hypermedia learning systems, with experts and novices showing different preferences to the use of hypermedia learning systems and requiring different levels of navigational support.

According to [26], in Web-based educational systems that consider either only learning styles or only cognitive traits, the relationship leads to more information. This additional information can be used to provide better adaptivity, for combined learning styles and cognitive traits instead of only for one of them. In systems that incorporate learning styles as well as cognitive traits, the interaction can be used to improve the detection process of the counterpart.

Reference [23] refers to the importance of the learning style as one of the learners' characteristic influencing positively on the group productivity and effectiveness.
Learners' personal features are taken into account by many researchers for forming learner groups. For example, reference [31] studied the impact of personality and group formation on the learner's performance. The information obtained by this study can be incorporated in collaborative systems as criteria for group formation, with the aim of favouring system situations in which learners are prone to get better results. According to [44], some dimensions of the learning style model seem to affect the quality of the resulting work.

Reference [69] point out that the most existing AEHSs utilize the learner models that take account of only a small number of learner characteristics and they were wondering if there is an AEHS that incorporates a multidimensional learner model to accommodate multiple learner characteristics, such as knowledge, learning styles, backgrounds, and preferences.

We conducted a review of the literature on learner characteristics used for different types of adaptation to existing AEHS so far. Table 1 presents the characteristics of students used by AEHS for different types of adaptation. The adaptive techniques commonly used are the curriculum sequencing, adaptive presentation, adaptive navigation and adaptive collaboration support, or adaptive group formation and/or peer help.

In Table 1, none of the AEHSs supports all the adaptation techniques mentioned above. In general, these systems use combinations of these to enrich adaptive functionality and enhance the support offered to learners. On the one hand, the majority of these systems supports adaptive navigation (thirty-three out of forty), which is one of the most popular techniques in current adaptive hypermedia systems, adaptive presentation (twenty-eight out of forty), and curriculum sequencing (twenty-three out of forty). On the other hand, some of these systems use adaptive collaboration support or adaptive group formation and/or peer help techniques (four out of forty). In the future, it will be a real challenge for designers to incorporate all adaptation techniques in the AEHSs.

Reference [11] statement is valid yet that adaptive navigation support is now a popular research area, while the adaptive presentation is the most popular and most studied hypermedia adaptation method.

The most of the AEHSs in Table 1 use one up to three learner characteristics for one up to three kinds of adaptation that usually are curriculum sequencing, adaptive presentation, and navigation. Seven out of forty of the AEHSs in Table 1 use four up to seven characteristics of adaptation, and only one out of forty, uses eight characteristics for adaptation. Also, a small amount of the AEHS in Table 1 support adaptive collaboration or adaptive group formation and/or peer help.

In Table 1, there are AEHSs that use one only learner characteristic for adaptation, such as the COFALE that uses the mental models for both adaptive presentation and adaptive collaboration, and the ALICE that uses the knowledge level for adaptive navigation.

There are AEHSs that use more than one learner characteristic for adaptation with the same technique as, 
for example, the ActiveMath uses for curriculum sequencing the preferences and cognitive goals, the MetaDoc uses for adaptive presentation the preferences, cognitive goals, and interests, while the AHA! uses for adaptive navigation the preferences, knowledge level, and cognitive style.

Table 1. Learner characteristics used for adaptation in the AEHSs

\begin{tabular}{|c|c|c|c|c|}
\hline AEHS & Curriculum Sequencing & Adaptive Presentation & $\begin{array}{c}\text { Adaptive Navigation } \\
\text { Support }\end{array}$ & $\begin{array}{c}\text { Adaptive } \\
\text { Collaboration } \\
\text { Support or Adaptive } \\
\text { Group Formation \& } \\
\text { Peer Help }\end{array}$ \\
\hline ACE [67] & interests, learning style & & knowledge level & \\
\hline ActiveMath [46] & cognitive goals, abilities & preferences, abilities & knowledge level & \\
\hline AES-CS [72] & & cognitive style & knowledge level & \\
\hline AHA! [20] & & $\begin{array}{l}\text { preferences, cognitive } \\
\text { style, knowledge level }\end{array}$ & $\begin{array}{l}\text { preferences, cognitive } \\
\text { style, knowledge level }\end{array}$ & \\
\hline ALE [65] & & $\begin{array}{c}\text { knowledge level, } \\
\text { preferences, learning style }\end{array}$ & $\begin{array}{c}\text { knowledge level, } \\
\text { preferences, learning style }\end{array}$ & \\
\hline ALICE [34] & & & knowledge level & \\
\hline Anatom-Tutor [7] & & knowledge level & & \\
\hline APELS [14] & & $\begin{array}{l}\text { ethos, learning goals and } \\
\text { prior knowledge }\end{array}$ & $\begin{array}{l}\text { ethos, learning goals and } \\
\text { prior knowledge }\end{array}$ & \\
\hline AST [66] & learning style & & knowledge level & \\
\hline CAMELEON [40] & & $\begin{array}{c}\text { knowledge level, learning } \\
\text { style }\end{array}$ & interactivity actions & \\
\hline COFALE [18] & & mental models & & mental models \\
\hline CS383 [16] & & learning style & & \\
\hline DEPTHS [33] & & $\begin{array}{c}\text { performance level \& } \\
\text { preferences }\end{array}$ & performance level & \\
\hline EDUCE [36] & & learning style & learning preferences & \\
\hline ELM-ART [13] & preferences & & knowledge level & \\
\hline eTeacher [61] & & learning style & learning style & \\
\hline Flexi-OLM [43] & & $\begin{array}{l}\text { preferences, learning \& } \\
\text { cognitive style }\end{array}$ & & \\
\hline Hypadapter [29] & & $\begin{array}{c}\text { knowledge level, } \\
\text { preferences, interests }\end{array}$ & experience & \\
\hline ILASH [4] & & $\begin{array}{c}\text { prerequisites, knowledge } \\
\text { level }\end{array}$ & learning style & \\
\hline INSPIRE [55] & $\begin{array}{l}\text { cognitive goals, } \\
\text { knowledge level }\end{array}$ & $\begin{array}{l}\text { learning style, interactivity } \\
\text { actions }\end{array}$ & knowledge level & \\
\hline InterBook [13] & cognitive goals, interests & & $\begin{array}{l}\text { knowledge level, } \\
\text { preferences }\end{array}$ & \\
\hline ISIS-Tutor [12] & $\begin{array}{c}\text { knowledge level, } \\
\text { experience }\end{array}$ & & $\begin{array}{l}\text { knowledge level, cognitive } \\
\text { goals }\end{array}$ & \\
\hline iWeaver [76] & & learning style & & \\
\hline KBS Hyperbook [50] & $\begin{array}{c}\text { cognitive goals, } \\
\text { prerequisites }\end{array}$ & & knowledge level & \\
\hline Knowledge-Sea II [1] & & & $\begin{array}{l}\text { user or user's group with } \\
\text { similar goals and } \\
\text { knowledge (social } \\
\text { navigation) }\end{array}$ & \\
\hline Lecomps [42] & $\begin{array}{l}\text { learning style, learning } \\
\text { goals, knowledge level. }\end{array}$ & & learning style. & \\
\hline LS-AEHS [48] & & $\begin{array}{c}\text { knowledge level and } \\
\text { learning style }\end{array}$ & $\begin{array}{c}\text { knowledge level and } \\
\text { learning style }\end{array}$ & \\
\hline LSAS [5] & & learning style & knowledge level & \\
\hline MANIC [68] & knowledge level & learning style preferences & & \\
\hline MetaDoc [8] & & $\begin{array}{c}\text { knowledge level, } \\
\text { preferences, interests }\end{array}$ & experience & \\
\hline MOT 2.0 [19] & & learning style & & $\begin{array}{c}\text { knowledge level, } \\
\text { social and grouping } \\
\text { features }\end{array}$ \\
\hline MyProject [54] & & & knowledge level & \\
\hline
\end{tabular}




\begin{tabular}{|c|c|c|c|c|}
\hline NetCoach [75] & knowledge level & & knowledge level & \\
\hline PCMAT [21] & & $\begin{array}{c}\text { knowledge level, learning } \\
\text { style, } \\
\text { learning rate }\end{array}$ & $\begin{array}{l}\text { learning style, learning } \\
\text { rate. }\end{array}$ & \\
\hline ProPer [35] & & $\begin{array}{c}\text { Knowledge level, learning } \\
\text { goals, learning style, } \\
\text { language, preferences, } \\
\text { disabilities. }\end{array}$ & $\begin{array}{l}\text { learning goals, interactivity } \\
\text { actions, learner's previous } \\
\text { and current knowledge }\end{array}$ & \\
\hline PUSH [30] & & learning goals & & \\
\hline TANGOW [15] & $\begin{array}{l}\text { learning style, activity } \\
\text { actions, preferences } \\
\text { about learning strategy, } \\
\text { language, and age }\end{array}$ & & $\begin{array}{c}\text { preferences about learning } \\
\text { strategy }\end{array}$ & $\begin{array}{l}\text { Users' personal } \\
\text { features, } \\
\text { preferences, } \\
\text { knowledge and } \\
\text { interactivity actions }\end{array}$ \\
\hline $\begin{array}{c}\text { TANGOW-WOTAN } \\
\text { [44] }\end{array}$ & $\begin{array}{c}\text { learning style, } \\
\text { interactivity actions, } \\
\text { preferences about } \\
\text { learning strategy, } \\
\text { language, and age }\end{array}$ & & $\begin{array}{c}\text { preferences about learning } \\
\text { strategy }\end{array}$ & cognitive style \\
\hline WELSA [57] & & preferences, learning style & preferences & \\
\hline WHURLE [47] & & learning style & $\begin{array}{c}\text { preferences, knowledge } \\
\text { level }\end{array}$ & \\
\hline
\end{tabular}

In Table 2, we can see the learner characteristics and the number of the AEHSs using them.

The learner characteristics used in the AEHSs in Table 1 are fifteen. The major learners' characteristics used in the AEHSs in Table 1 are the knowledge level (twentyseven out of forty) and learning or cognitive style (twenty-four out of forty). The preferences and learning or cognitive goals used in the AEHSs in Table 1 are eleven and seven out of forty, respectively, while the other characteristics used in a small amount of the AEHSs. Thus, the most popular and usable learner characteristics for adaptation support in the AEHSs are the knowledge level, learning or cognitive style, preferences, and learning or cognitive goals.

Table 2. Learner characteristics used by the AEHSs in Table 1

\begin{tabular}{|c|c|c|c|c|c|}
\hline Learner characteristic & $\begin{array}{c}\text { Number of } \\
\text { AEHSs using } \\
\text { them }\end{array}$ & $\begin{array}{c}\text { Curriculum } \\
\text { Sequencing }\end{array}$ & $\begin{array}{c}\text { Adaptive } \\
\text { Presentation }\end{array}$ & $\begin{array}{c}\text { Adaptive } \\
\text { Navigation } \\
\text { Support }\end{array}$ & $\begin{array}{c}\text { Adaptive Collaboration Support } \\
\text { or Adaptive Group Formation \& } \\
\text { Peer Help }\end{array}$ \\
\hline Knowledge level & 27 & 5 & 9 & 17 & 1 \\
\hline Learning or cognitive style & 24 & 5 & 18 & 5 & 1 \\
\hline Preferences & 11 & 3 & 10 & 6 & 0 \\
\hline Learning or cognitive goals & 7 & 5 & 3 & 3 & 1 \\
\hline Interactivity actions & 4 & 1 & 1 & 2 & 0 \\
\hline Experience & 3 & 1 & 1 & 2 & 0 \\
\hline Interests & 3 & 2 & 2 & 0 & 0 \\
\hline Language & 3 & 2 & 1 & 0 & 0 \\
\hline Abilities - disabilities & 2 & 1 & 2 & 0 & 0 \\
\hline Prerequisites & 2 & 1 & 1 & 0 & 1 \\
\hline Prior or previous knowledge & 1 & 0 & 1 & 1 & 0 \\
\hline Performance level & 1 & 0 & 1 & 1 & 0 \\
\hline Mental models & 1 & 0 & 1 & 0 & 0 \\
\hline Learning rate & 1 & 0 & 1 & 1 & 0 \\
\hline Ethos & 1 & 0 & 1 & 1 & \\
\hline
\end{tabular}

Regarding the four key features, we concluded that:

Most of the AEHS (17 out of 27) use the learner's level of knowledge as an adaptation feature for adaptive navigation technique.

Most of the AEHS (18 out of 24) use the learner's learning or cognitive style as an adaptive feature of adaptive presentation technique.

Most of the AEHS (10 out of 11) use the learner's preferences as an adaptive feature of adaptive presentation technique.

Most of the AEHS (5 out of 7) use the learner's learning or cognitive goals as an adaptive feature for curriculum sequencing technique.

\section{THE CASE OF THE AEHS MATHEMA}

The Web-based AEHS MATHEMA is a learning system that dynamically generates courses in physics. The general pedagogical aim of the MATHEMA is to support senior high school learners or novices of higher education, through an interactive and constructive 
educational material, in learning physics individually and/or collaboratively, and overcoming their possible misconceptions and learning difficulties.

In Fig. 1, we can see a snapshot of the user interface of the MATHEMA. MATHEMA combines both the constructivism and socio-constructivism didactic models, and it also supports both individual and collaborative learning. In the MATHEMA through constructivism, the learner facilitated by having him/her find topics or issues, find resources, plan investigations and activities, practice self-evaluation and formulate principles. The learning process involves the learner in conceptually constructing of meanings from what demonstrated or experienced.

The learning process involves the learner in conceptually constructing of meanings from what demonstrated or experienced. In the design of interactive educational material, a more enriched learning experience occurs when both content and teaching style adapted to the learners with different learning styles.

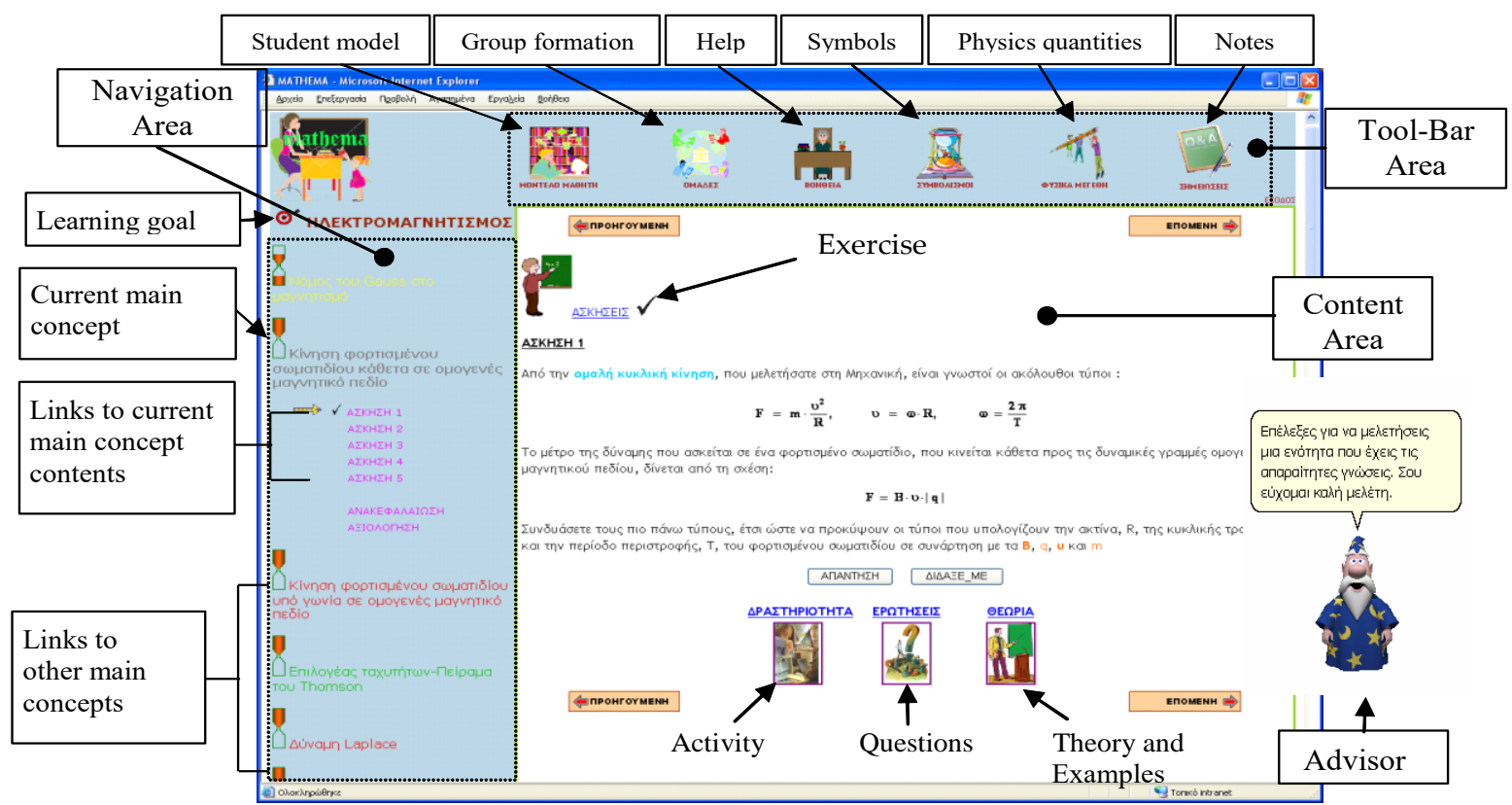

Fig.1. A snapshot of the user interface of the MATHEMA

Design is an active process that influences society, creating its material culture [58]. The design of the MATHEMA aims at developing an effective system based on the constructivism and socio-cultural perceptions of learning (student-centred) in such a way as to use the necessary and proper intelligent and adaptive techniques, which one modern AEHS should have, as well as its functions should have high functionality, usability, and usefulness.

The innovative techniques, making the MATHEMA distinguish from other AEHSs are the following:

- The meta-adaptation technique that assists the learner to choose the most appropriate navigation technique among the four offers (direct guidance, link annotation, link hiding, and link sorting) that suits his or her profile taking into account his or her Web experience and the level of knowledge in the current learning goal.

- The interactive problem-solving technique through activities by using modern didactic approaches and combining both individual and collaborative learning. It is important for physics learning.

- The adaptive group formation and peer help technique assisting the learner in selecting of the most proper collaborator from a list of candidate collaborators that the system creates by taking into account the learner's learning style, and the learning style and knowledge level on the current learning goal of candidate collaborators.

- The learner characteristics we use of the various kinds of adaptation.

When we planned the MATHEMA, first we decided to select the supported intelligent and adaptation techniques. The learning theories and didactic strategies are factors of decisive importance for selecting the adaptive and intelligent techniques would be used in developing an AEHS.

Modern adaptive and intelligent techniques needed in supporting didactic approaches such as problem-solving, explorations, and others should use the learner-centered design (LCD) principles [63].

The domain of application in an AEHS may play the most important role in selecting of adaptive and intelligent techniques as well as the chosen dimensions of students' learning style for group formation should fit with the domain of application [53].

The AEHSs developed so far using combinations of adaptive and intelligent techniques to increase their functionality. 
After much contemplation, we selected the following techniques: curriculum sequencing, adaptive presentation, adaptive, and meta-adaptive navigation support, interactive problem-solving support, and the adaptive group formation and peer helping.

In order to select the learner's characteristics being used in the above techniques, we took into account all the suggestions mentioned above and especially the suggestions of:

(a) Reference [69] that the learner characteristics being considered in the AEHS are knowledge level, learning styles, experience, background, and preference.

(b) Reference [44] that some dimensions of the learning style model seem to affect the quality of the resulting work.

(c) Reference [17] that many studies have found that learners with different levels of prior knowledge benefit differently in hypermedia learning systems, with experts and novices showing different preferences to the use of hypermedia learning systems and requiring different levels of navigational support.

Also, we took into account the findings of the abovementioned literature review, that is, the most popular and usable learner characteristics for adaptation support in the AEHSs are the knowledge level, learning or cognitive style, preferences, and learning or cognitive goals. Moreover, a learning style model adopted by all recent AEHSs, and it has been immediately connected with the learning theories and didactic approaches them based upon. In the selection of the proper student characteristics for adaptive support, we took note of the following.

Not all learners can manage the high level of control offered by hypermedia systems. Some learners may lose or become disoriented in such systems [51], and a number of studies show that learners' prior knowledge is an essential factor that influences the degree of disorientation that learners experience in hypermedia systems. Also, [13] has shown that the adaptive navigation support techniques link annotation, link sorting, and link hiding can improve learner performance in hypermedia by significantly reducing navigation difficulty. The purpose of meta-adaptation is to help the learner to take decisions for the navigation technique that fits best for him or her. That is, through the metaadaptation, we intended to help the learners be more selfregulated learners. Different kinds of tasks are typically involved in collaborative learning activities. One of them, eventually the most eminent, is problem-solving. Much of the current work in cognitive psychology has shown that students learn better when engaged in solving problems [45].

After an extended research [51] we decided to design and implement the learner-controlled adaptive group formation technique in the AEHS MATHEMA by using the abstract and concrete dimensions of learning styles and knowledge level on the current learning goal of learners as characteristics for adaptation. Consequently, the learning style model is a factor of decisive importance when designing modern educational systems.

Therefore, the learner characteristics we decided to select, for various kinds of adaptation, are the following: learning goal, knowledge level for each main concept, knowledge level on each learning goal, Web experience, learning style, abstract or concrete dimension of learning style, preference for visual and/or verbal feedback, preference for the kind of navigation, preference of using or not the navigation guidance and prerequisites.

In the following paragraphs, we describe how adaptation techniques use the above learner characteristics to adapt to the learner the content, link structures, and peers for forming groups.

Curriculum sequencing: The curriculum sequencing in the MATHEMA is based upon the Web experience and knowledge level on the current learning goal of the learner. The main concepts of learning goal in the MATHEMA are progressively presented according to the internal structure of the concepts. The concepts of the learning goal are organized in a layered structure following a simple-to-complex sequence [59], according to which, in the first layer the simplest and most fundamental concepts included, providing an overview of the learning goal, and then, next layers of concepts add complexity or detail to a part or aspect of the learning goal [55].

Curriculum sequencing is used in combining with the link annotation technique in the case that the learner at the first time he or she log into the system declares that he or she has little or no Web experience and medium knowledge level on the current learning goal. In this case, the main concepts of the learning goal are presented in three layers. In the first level, the main concepts with a low degree of difficulty presented. At the second level, the main concepts with a medium degree of difficulty presented, and in the third level, the main concepts with a high degree of difficulty presented. In order to have the learner study the main concepts of the next level, must study the main concepts of the current level.

Adaptive presentation: The most effective learners should use multiple strategies to make sure that they check their comprehension. The goal is for the student to develop self-awareness of his or her comprehension [4]. Thus, we need adequate didactic strategies to promote meaningful learning. If the learners are able to decide their own pathway in selecting the available information, in the way that best suits their own learning style, and then the learning process perhaps is more efficient [9]. The adaptive presentation in the MATHEMA is based upon the Kolb's learning style model [38]. Reference [37] argues that most people prefer to learn from one of the following four learning styles: Diverging, Assimilating, Converging and Accommodating. Taking into consideration the Kolb's learning style model and the researches of [27] and [70], as well as our research [52], we consider that the most proper sequence of didactic strategies matching for each of learners' learning style for adaptive presentation is: 
(1) Diverging: (a) Questions, Demonstrations; (b) Presentation of Theory and Examples; (c) Exercise Solving; (d) Problem Solving Activity.

(2) Assimilating: (a) Presentation of Theory and Examples; (b) Exercise Solving; (c) Problem Solving Activity; (d) Questions, Demonstrations.

(3) Converging: (a) Exercise Solving; (b) Problem Solving Activity; (c) Questions, Demonstrations; (d) Presentation of Theory and Examples.

(4) Accommodating: (a) Problem Solving Activity; (b) Questions, Demonstrations; (c) Presentation of Theory and Examples; (d) Exercise Solving.

Adaptive navigation support: The adaptive navigation support in the MATHEMA is based upon the learner's Web experience and knowledge level on the learner's current learning goal. At the first time that a learner logs into the system, he or she declares his/her Web experience and knowledge level on the current learning goal. After the learner's declaration, the system adapts the proper navigation technique to him or her according to his or her Web experience and knowledge level on the current learning goal. Thus, the adaptive navigation support in the MATHEMA helps the learners avoid the lost in hypermedia syndrome by offering them the following techniques:

Direct guidance: The system suggests the next unit be studied by the learner regardless of his or her performance in earlier units. In the Navigation Area, a "next unit" link is presented. The content to be learned by learners is adapted to each individual learner according to his or her learning style. The units are presented according to their level of difficulty. Initially, the low degree of difficulty unit is presented, and at the end, the higher degree of difficulty unit is presented.

Link Annotation: The system colors the visible links to the units as follows:

a) The link to the current unit that the learner is studying is colored grey and it is boldfaced.

b) The links to the units that the learner is not ready to study are coloured red.

c) The links to the units that the learner has already studied and has succeeded in the assessment tests are coloured black.

d) The links to the units that the learner is ready to study are coloured as follows:

- Dark green if a unit has a high degree of difficulty.

- Green if a unit has a medium degree of difficulty.

- Light green if a unit has a low degree of difficulty.

After of each learner's assessment, the system revises the link colour. A red link to a unit turns green when the learner has successfully assessed for all the prerequisite units.

Apart from the links to the units, there are links to pages (content) of a unit (local navigation). The links to pages of a unit are different for different teaching methods. Links to pages of a unit are coloured magenta.
An animated arrow in front of the link denotes the current page (see Fig. 1).

Link hiding: Initially, the system presents the low degree of difficulty units only. If the learner succeeds in the assessment tests for all the low degree of difficulty units, then the system will present the medium degree of difficulty units. If the learner succeeds in the assessment tests for all the medium degree of difficulty units, then the system will present the highest degree of difficulty units. The least knowledge level of a learner to succeed in the assessment tests is decided by the system administrator in a similar way we mentioned above.

Link sorting: The system sorts the units according to their level of difficulty. The lowest degree of difficulty units is listed at the top of the list. The medium degree of difficulty units is listed in the middle of the list. The highest degree of difficulty units is listed at the bottom of the list.

Moreover, except the above techniques, the system uses an "Advisor" advising the learner in his/her navigation.

Meta-adaptive navigation support: The meta-adaptive navigation support in the MATHEMA is based upon the learner's Web experience and knowledge level on the learner's current learning goal. The meta-adaptive navigation is a learner-controlled meta-adaptation (metaadaptability) technique where the system assists the learner in decision-making for the navigation technique that best suits to him or her by presenting to him or her the advantages and disadvantages of the navigation support techniques and additional information about them when the learner fulfills the requirements for metaadaptation. As we mentioned above, for the first time that a learner logs into the system, he or she declares to the system his or her Web experience and knowledge level on the current learning goal. After the learner's declaration, the system adapts the proper navigation technique to him or her according to his or her Web experience and knowledge level on the current learning goal. The learner informed by the system about the navigation technique adapted to him or her and it also explains the reasons why the automatically selected by the system navigation technique adapted to him or her.

However, the system recommends the learner changing the automatically selected navigation technique, when he or she has fulfilled the requirements to ask different navigation technique (successful assessments on $\boldsymbol{n}$ main concepts). The $\boldsymbol{n}$ defined by the educator. The system also informs the learner that, if he or she does not wish to follow its automatically selected navigation technique and recommendation, then he or she can change the navigation technique when he or she wishes through his or her Student Model on the Tool-Bar Area.

Whenever the learner has fulfilled the requirements for meta-adaptation, then the meta-adaptive mechanism will appear information with the advantages and disadvantages and other relevant information for all the four navigation techniques on the screen and the learner is able to select one that suits him or her best.

Interactive problem-solving support: For the 
interactive problem-solving support in the MATHEMA we do not use any learner characteristic for adaptation. For this technique, the system uses a general framework of problem-solving activity. The framework includes six steps:

Step 1: Activation of prior knowledge (use of formulas from previous knowledge and the formulas of the subject matter that the learners are studying with the aim of extracting formulas for calculating certain physical quantities (or dimensions)).

Step 2: Definition of restrictions on the parameters' values of resulting formulas in Step 1 through a guided dialog with the system.

Step 3: Application of formulas on real values of parameters, and prediction of the kind of motion in each case.

Step 4: Work with the simulation, comparison of the results resulting from the simulation to the results of Step 3 and explanation of the differences.

Step 5: Negotiation and Collaboration in pairs of learners to share their experience, opinions, findings, etc. for co-constructing knowledge.

Step 6: Checking of the results through a guided dialog with the system. The aim of the guided dialog is to detect either possible misconceptions or learning difficulties of the learners to help them reflect and reconstruct their own cognitive model.

Adaptive group formation and peer help: The learnercontrolled adaptive group formation and peer help technique in the MATHEMA is based upon the abstract or the concrete dimension of the learner learning style and his or her candidate peers' learning style and knowledge level on the current learning goal.

The adaptive group formation technique of the MATHEMA is an innovative technique because the AEHSs developed so far, are based upon a systemcontrolled and/or upon an educator-controlled design, that is, the group formation is decided by the system or by the educator and the learners are only informed without allowing them to change it or negotiate a collaboration agreement with their possible candidate peers [53].

The adaptive group formation and peer help implemented in the MATHEMA operates as follows: When a learner requests for collaborators then the system creates a priority list of possible candidate peers for a certain learner, taking into account the abstract or the concrete dimension of his or her learning style and his or her candidate peers' learning style and knowledge level on the current learning goal as well. They are sorted according to their learning style and, if some of them belong to the same learning style, then they are sorted according to their knowledge level on the current learning goal. Also, the system informs the learner that his or her most significant possible candidate peer is at the top of the list and the least significant is at the bottom of the list. After this, the learner chooses his or her peer and negotiates a collaboration agreement with him or her.

\section{EVALUATION OF THE MATHEMA}

In the frame of the summative evaluation of the AEHS MATHEMA, we conducted a research to investigate the usability, usefulness, and appropriateness of the system's functions, as well as to detect problems in the system functionality or interface, taking into account the usercentred evaluation of adaptive and adaptable systems, as it describe the [74]. User-centred evaluation can serve three goals: verifying the quality of a product, detecting problems and supporting decisions. According to [74], questionnaires are the most common evaluation method in the studies, and one of the advantages of questionnaires is the large number of participants that can be accommodated (compared with interviews or other methods). Also, [74] in their review on user-centred evaluation used variables, concluded that the usability has been the most often measured characteristic, followed by perceived usefulness and appropriateness of adaptation. Such variables are suitable for quantitative measurement, using Likert-type scales [41], for example.

\section{A. Participants}

The research was conducted in the Department of Informatics and Telecommunications, University of Athens, Greece. Forty-three (43) undergraduate students participated in the research.

\section{B. Experimental Procedure}

The evaluation of the AEHS MATHEMA was done by the students in the frame of the discipline "Informatics and Education". It was given as individual work to the participants, and it was graded with $60 \%$ of the total grade. At the beginning, we gave the learner information about all the functions of the MATHEMA and a demonstration of them was done on the system. The learners studied the environment of the MATHEMA, examined all the functions, and were asked to work with the system on specific activities and educational material to evaluate all the functions. At the end, they completed the questionnaire. The evaluation lasted 30 days.

\section{Method}

Our evaluation approach was based upon the [74] conclusion about the user-centred evaluation we mentioned above. Thus, we chose the questionnaire as the method of user-centred evaluation of our system. During the design of the questionnaire for the system's evaluation, we also took into account the [32] guidelines for specifying and measuring usability. The Likert-type scale has five categories (not at all satisfied, slightly dissatisfied, neutral, very satisfied, and extremely satisfied). The characterization "not at all satisfied" is coded to -2 , the characterization "slightly satisfied" is coded to -1 , the characterization "neutral" is coded to 0 , the characterization "very satisfied" is coded to 1 , and the characterization "extremely satisfied" is coded to 2 .

\section{Data Collection}


The data collection was done with the evaluation questionnaire completed by all (43) of the participants.

The answers of the participants about the questions, graded on the Likert-type scale, were grouped into two categories, as follows: "Dissatisfaction/Neutral" (not at all satisfied, slightly dissatisfied, and neutral), and "Satisfaction" (very satisfied, extremely satisfied).

In Table 3, a number of the participants for each the two categories have listed.

\section{E. Data Analysis and Results}

For the data analysis, the Yates' chi-square statistic method was used. The null hypothesis $\left(\mathrm{H}_{0}\right)$ was that the amounts of the two categories (Dissatisfaction/Neutral, Satisfaction) were equal (they have no statistically significant relationship).

In Table 4, the results of the quantitative data analysis are presented.

Table 3. Processing and classification of data

\begin{tabular}{|c|c|c|c|}
\hline Function & Ratings & $\begin{array}{c}\text { Dissatisfaction/ } \\
\text { Neutral }\end{array}$ & Satisfaction \\
\hline \multirow[t]{2}{*}{ Adaptive presentation } & usefulness & $3(7 \%)$ & $40(93 \%)$ \\
\hline & usability & $6(14 \%)$ & $37(86 \%)$ \\
\hline Adaptive navigation techniques & usability & $4(10 \%)$ & $39(90 \%)$ \\
\hline Meta-adaptive navigation technique & usefulness & $5(12 \%)$ & $38(88 \%)$ \\
\hline Adaptive group formation technique & usability & $18(42 \%)$ & $25(58 \%)$ \\
\hline \multirow[t]{2}{*}{ Feedback } & usefulness & $3(7 \%)$ & $40(93 \%)$ \\
\hline & usability & $3(7 \%)$ & $40(93 \%)$ \\
\hline Learner control and freedom & usability & $5(12 \%)$ & $38(88 \%)$ \\
\hline Flexibility and Efficiency of Use & & $2(5 \%)$ & $41(95 \%)$ \\
\hline
\end{tabular}

Table 4. Evaluation of the main functions of the AEHS MATHEMA

\begin{tabular}{|c|c|c|c|}
\hline Function & Ratings & $\mathrm{x}^{2}$ & $\mathrm{p}$ \\
\hline \multirow[t]{2}{*}{ Adaptive presentation } & usefulness & 31.84 & $2.41 \mathrm{E}-08$ \\
\hline & usability & 22.35 & $2.93 \mathrm{E}-06$ \\
\hline \multirow[t]{2}{*}{ Adaptive navigation techniques } & usefulness & 41.023 & $3.2 \mathrm{E}-11$ \\
\hline & usability & 26.88 & $1.3 \mathrm{E}-07$ \\
\hline \multirow[t]{2}{*}{ Meta-adaptive navigation technique } & usefulness & 25.32 & $6.45 \mathrm{E}-07$ \\
\hline & usability & 22.35 & $2.93 \mathrm{E}-07$ \\
\hline \multirow[t]{2}{*}{ Adaptive group formation technique } & usefulness & 28.49 & $1.3 \mathrm{E}-07$ \\
\hline & usability & 1.14 & 0.28 \\
\hline \multirow[t]{2}{*}{ Feedback } & usefulness & 31.84 & $2.41 \mathrm{E}-08$ \\
\hline & usability & 31.84 & $2.41 \mathrm{E}-08$ \\
\hline Learner control and freedom & usability & 25.32 & $6.45 \mathrm{E}-07$ \\
\hline \multicolumn{2}{|l|}{ Flexibility and Efficiency of Use } & 33.58 & 4.07E-09 \\
\hline
\end{tabular}

\section{F. Discussion and Conclusion}

\section{Analysis of the results}

According to the results of Table 4, the null hypothesis, where the amounts of the two categories (Dissatisfaction/Neutral, Satisfaction) are assumed as equal, is rejected (all the $\mathrm{p}$ values are less than 0.05 ) for the usefulness and usability of almost all the functions listed in Table 4, except of the usability of the adaptive group formation technique $(\mathrm{p}=0.28>0.05)$.

The evaluation of the adaptive presentation of content showed that a lot of the participants $(93 \%)$ consider it as useful $(\mathrm{p}=2.41 \mathrm{E}-08<0.05)$ and a lot of the participants $(86 \%)$ consider it as usable $(\mathrm{p}=2.93 \mathrm{E}-06<0.05)$.

The evaluation of the adaptive navigation techniques showed that all of the participants $(100 \%)$ consider it as useful $(\mathrm{p}=3.2 \mathrm{E}-11<0.05)$ and a lot of the participants $(90 \%)$ consider it as usable $(\mathrm{p}=1.3 \mathrm{E}-07<0.05)$.

The evaluation of the meta-adaptive navigation technique showed that a lot of the participants $(88 \%)$ consider it as useful $(\mathrm{p}=6.45 \mathrm{E}-07<0.05)$ and a lot of the participants $(86 \%)$ consider it as usable $(p=2.93$ E$07<0.05)$.

The evaluation of the adaptive group formation technique showed that a lot of the participants $(91 \%)$ consider it as useful $(\mathrm{p}=1.3 \mathrm{E}-07<0.05)$ and a lot of the participants $(58 \%)$ consider it as no usable $(\mathrm{p}=0.28>0.05)$.

The evaluation of the feedback showed that a lot of the participants $(93 \%)$ consider it as useful $(p=2.41 \mathrm{E}-$ $08<0.05)$ and usable $(\mathrm{p}=2.41 \mathrm{E}-08<0.05)$.

The evaluation of the learner control and freedom showed that a lot of the participants $(88 \%)$ consider it as usable $(\mathrm{p}=6.45 \mathrm{E}-07<0.05)$.

The evaluation of the flexibility and efficiency of use of the MATHEMA showed that a lot of the participants $(95 \%)$ consider it as flexible and efficient $(\mathrm{p}=4.07 \mathrm{E}-$ $09<0.05)$.

\section{Other remarks}

In general, the functions of the AEHS MATHEMA are 
distinguished for their high functionality, usefulness, and usability, as it is mentioned by the participants.

Moreover, a research for the achievement of the learners when they study a subject matter through the MATHEMA [51] showed that there was a significant difference in learning performance of the students from the pre-test to the post-test $(\mathrm{F}=53.45, \mathrm{p}=0.0184<0.05)$ performed on the same subject matter.

The comments and the proposals of the participants about the weaknesses of the system, on the collaboration help through the chat tool of the MATHEMA and the "assessment tool", were taken into consideration by the authors for the improvement these functions.

After this first essay, considering that the usability of the "adaptive group formation tool" was not satisfactory enough, we improved the adaptive group formation technique to be more usable. After a detailed analysis of the answers of the participants to the open-ended questions, we concluded that the cause of the inadequacy was lying in the usage made by many of the participants of the negotiation protocol via the "chat tool". In order to solve this usability problem, we make use of an "Advisor" to help the learners in following appropriately the negotiation protocol and giving the suitable information about their collaboration. Also, we improved the functionality of the "chat tool".

\section{SUMmARY AND FUtURE WORK}

Taking into consideration that the learner characteristics play an important role in the development of AEHSs, we took a literature review about the learner characteristics used for various kinds of adaptation in existing AEHSs so far. The literature review showed that the major characteristics of learners used in AEHSs so far are the knowledge level and learning or cognitive style. Also, the literature review showed that the most of the AEHSs use one up to three learner characteristics for one up to three kinds of adaptation that usually are curriculum sequencing, adaptive presentation, and adaptive navigation. One only system uses eight characteristics for adaptation. Also, a small amount of the AEHSs support adaptive collaboration or adaptive group formation and/or peer help.

The MATHEMA system goes a step forward: In the aim to better support the learning process, it enriches the adaptive and intelligent functionality of the AEHSs, by implementing and exploiting the following techniques: curriculum sequencing, adaptive presentation, adaptive and meta-adaptive navigation support, interactive problem-solving support, and the adaptive group formation and peer helping.

In order to support the above techniques, we selected the following learner characteristics for adaptation: learning goal, knowledge level for each main concept, knowledge level on each learning goal, Web experience, learning style, abstract or concrete dimension of learning style, preference for visual and/or verbal feedback, preference for the kind of navigation, preference of using or not the navigation guidance and prerequisites.
The summative evaluation of the MATHEMA showed that almost all of its functions are easily usable by the learner and almost all of them are proven to be very effective in the learning process. In conclusion, we consider that the choice of the learner characteristics that we have performed and their usage in the adaptation and intelligent techniques we developed in the MATHEMA, is successful.

Moreover, this paper answers to the [64] question about the being of an AEHS that incorporates a multidimensional learner model to accommodate multiple learner characteristics, such as knowledge, learning styles, backgrounds and preferences, as well as to [24] question on which educational process should be adapted an AEHS.

In our future plans, we intend to improve the adaptive group formation algorithm. Our aim is to formulate a more extended algorithm able to deal with groups of students which follow a different curriculum. Also, we have addressed an experimental study to investigate how much the proposed learner-centred meta-adaptation helps the students to take control over their own learning, so that to assume responsibility for their own cognitive development.

\section{REFERENCES}

[1] J. R. Ahn Farzan, \& P. Brusilovsky. "Social Search in the Context of Social Navigation". Journal of the Korean Society for Information Management. vol. 23, no 2, pp. 147-165, 2006.

[2] M. Alshammari, P. Anane \& R.J. Hendley. "Design and Usability Evaluation of Adaptive e-learning Systems Based on Learner Knowledge and Learning Style". In Proceedings of the Conference on Human-Computer Interaction--INTERACT 2015. pp. 584-591. Springer, 2015.

[3] M.T. Alshammari. "Adaptation based on Learning Style and Knowledge Level in E-Learning Systems". A thesis submitted to the University of Birmingham for the degree of Doctor of Philosophy, 2016.

[4] N. Bajraktarevic, W. Hall, \& P. Fullick. "ILASH: Incorporating Learning Strategies in Hypermedia". In Proceedings of the Fourteenth Conference on Hypertext and Hypermedia, 2003.

[5] N. Bajraktarevic, W. Hall, \& P. Fullick. "Incorporating Learning Styles in Hypermedia Environment: Empirical evaluation". In Proceedings of the AH2003 Workshop Adaptive Hypermedia and Adaptive Web-Based Systems (pp. 41-52), 2003.

[6] A. Battou, A. EL Mezouary, C. Cherkaoui, \& D. Mammas. "The Granularity Approach of Learning Objects to Support Adaptibility in Adaptive Learning Systems". Journal of Theoretical and Applied Information Technology, vol. 18, no 1, 2010.

[7] Beaumont. "User modelling in the interactive anatomy tutoring system ANATOM-TUTOR". User Modelling and User Adapted Interaction, vol. 4, no.1, 21-45, 1994.

[8] C. Boyle, \& A.O. Encarnacion. "MetaDoc: An Adaptive hypertext Reading System". User Modelling and User Adapted Interaction, vol. 4, no 1, pp. 1-19, 1994.

[9] C. Brickell. "Navigation and Learning", Australian Journal of Educational Technology, vol. 9, no. 2, pp. 103114, 1993.

[10] P. Brusilovsky, \& E. Milan. "User models for adaptive 
hypermedia and adaptive educational systems". In Brusilovsky P., Kobsa A., \& Nejdl W. (Eds.), The adaptive Web. Methods and strategies of Web personalization. LNCS 4321 (pp. 3-53). Berlin Heidelberg: Springer-Verlag, 2007.

[11] P. Brusilovsky. "Adaptive Navigation Support in Educational Hypermedia: The Role of Student Knowledge Level And The case For Meta-Adaptation". British Journal of Educational Technology, vol. 34, no 4, pp. 487-497, 2003.

[12] P. Brusilovsky, \& L. Pesin. "ISIS-Tutor: An adaptive hypertext learning environment". In Proceedings of the Japanese CIS Symposium on knowledge-based software engineering (JCKBSE’94). pp. 83-87, 1994.

[13] P. Brusilovsky, E. Schwarz, \& G. Weber. "A Tool for Developing Adaptive Electronic Textbooks on WWW". In Proceedings of the WebNet'96 conference. pp. 64-69, 1996.

[14] J. Canavan. Personalized E-Learning Through Learning Style Aware Adaptive Systems. Master's thesis, University of Dublin, Ireland, 2004.

[15] R.M. Carro, E. Pulido, \& P. Rodríguez. "TANGOW: A Model for Internet based Learning". Continuing Engineering Education and Life-Long Learning, vol. 11, no. $1-2,2001$

[16] C.A. Carver, R.A. Howard, \& W.D. Lane. "Enhancing student learning through hypermedia courseware and incorporation of student learning styles". IEEE Transactions on Education, vol. 42, no 1, pp. 33-38, 1999.

[17] C. Chen. "Intelligent web-based learning system with personalized learning path guidance", Computers \& Education, vol. 51, Issue 2, pp. 787-814, 2008.

[18] V. M. Chieu. "COFALE: An Authoring System for Supporting cognitive Flexibility". In Proceedings of the $6^{\text {th }}$ IEEE International Conference on advanced Learning Technologies (pp.335-339), 2007.

[19] A. I. Cristea, \& F. Ghali. "Towards Adaptation in Elearning 2.0". New Review of Hypermedia and Multimedia, vol. 17, no 2, pp. 199-238, 2011.

[20] P. De Bra, \& L. Calvi. "AHA! An Open Adaptive Hypermedia Architecture". New Review of Hypermedia and Multimedia, vol. 4, pp. 115-139, 1998.

[21] M. Fernandes, P. Couto, C. Martins, L. Faria, C. Bastos, \& F. Costa. "Learning Objects Recommendation in an Adaptive Educational Hypermedia System. Technology Innovations in Education". In Proceedings of the $8^{\text {th }}$ WSEAS International Conference on Educational Technologies (EDUTE '12), 2012.

[22] R. Flores, F. Ari, F. A. Inan, \& I. Arslan-Ari. "The Impact of Adapting Content for Learners with Individual Differences". Educational Technology \& Society, vol. 15, no. 3, pp. 251-261, 2012.

[23] B. S. Gardner, \& S. J. Korth. "Classroom strategies that facilitate transfer of learning to the workplace". Journal of Innovative Higher Education, vol. 22, no. 1, pp. 45-60, 1997.

[24] P. Germanakos, \& M. Belk. "The E-Learning Case. Human-Centred Web Adaptation and Personalization: From Theory to Practice". Human-Computer Interaction Series. Springer, 2016.

[25] A. P. Gilakjani. "The Significant Role of Multimedia in Motivating EFL Learners' Interest in English Language Learning". International Journal of Modern Education and Computer Science (IJMECS), vol.4, no.4, pp.57-66, 2012.

[26] S. Graf, \& Kinshuk. Learner Modelling Through Analyzing Cognitive Skills and Learning Styles. In H. H. Adelsberger, Kinshuk, J. M. Pawlowski, D. Sampson,
Handbook on Information Technologies for Education and Training (2nd edition), Springer, Heidelberg, pp. 179194, 2008.

[27] J.N. Harb, P.K. Hurt, R.E. Terry, \& K.J. Williamson. Teaching through the Cycle: Application of Learning Style Theory to Engineering Education at Brigham Young University, Provo, UT: Brigham Young University Press, 1995.

[28] N. Henze, \& W. Nejdl. "Adaptivity in the KBS Hyperbook System". In: P. Brusilovsky, P.D. Bra and A. Kobsa (eds.) Proceedings of Second Workshop on Adaptive Systems and User Modelling on the World Wide Web, Toronto and Banff, Canada, May 11 and June 23-24, 1999 Published as Computer Science Report, No. 99-07, Eindhoven University of Technology, Eindhoven. pp. 67-74, 1999.

[29] H. Hohl, H.-D. Böcker, \& R. Gunzenhäuser. "Hypadapter: An adaptive hypertext system for exploratory learning and programming". User Modelling and User-Adapted Interaction, vol. 6, no. 2-3, pp. 131-156, 1996.

[30] K. Höök, J. Karlgren, A. Waern, N. Dahlboack, C. Jansson, K. Karlgren, \& B. Lemaire. "A glass box approach to adaptive hypermedia". User Modelling and User-Adapted Interaction, vol. 6, no. 2-3, pp. 157-184, 1996.

[31] V.S. Hórreo, \& R.M. Carro. "Studying the impact of personality and group formation on learner performance". Groupware: Design, implementation, and use (pp. 287294). Springer, 2007.

[32] ISO 1993. Usability and ISO Standards.

[33] Z. Jeremic, J. Jovanovic, \& D. Gasevic. "Evaluating an Intelligent Tutoring System for Design Patterns: the DEPTHS Experience". Educational Technology \& Society, vol. 12, no. 2, pp. 111-130, 2009.

[34] A. Kavcic, M. Privosnik, Marolt, \& S. Divjak. "Educational hypermedia system ALICE: an evaluation of adaptive features". In proceedings of the WSEAS conference on Advances in multimedia, video and signal processing systems. pp. 71-76, 2002.

[35] I. Kazanidis \& M. Satratzemi. "Efficient authoring of SCORM courseware adapted to user learning style: the case of ProPer SAT". In M. Spaniol, Q. Li, R. Klamma \& R. Lau (eds.), LNCS, 5686, 196-205, 2009.

[36] D. Kelly \& B. Tangney. "Matching and Mismatching Learning Characteristics with Multiple Intelligence Based Content". In Proceedings of the $12^{\text {th }}$ International Conference on Artificial Intelligence in Education, AIED'05, pp. 354-361, 2005.

[37] D.A. Kolb. Experiential Learning: Experience as the Source of Learning and Development. Prentice-Hall, Inc. N.J: Englewood Cliffs, 1984.

[38] A.Y. Kolb, \& D.A. Kolb. The Kolb's Learning Style Inventory - Version 3.1, Technical Specifications, 2005.

[39] M. Kravcik, R. Klemke, L. Pesin, R. Hüttenhain, \& M. Specht. "Adaptive Learning Environment in WINDS". In P. Barker \& S. Rebelsky (Eds.), Proceedings of EdMedia: World Conference on Educational Media and Technology (pp. 1846-1851), 2002.

[40] M. Laroussi \& M. Ben Ahmed. "Providing an adaptive learning through the Web case of CAMELEON". In Proceedings of the fourth International CALISCE'98 conference on Computer Aided Learning and Instruction in Science and Engineering (pp. 411-416), 1998.

[41] R. Likert. "A Technique for the Measurement of Attitudes". Archives of Psychology, 140, pp. 1-55, 1932.

[42] C. Limongelli, F. Sciarrone, M. Temperini, \& G. Vaste. "Adaptive learning with the LS-Plan system: a field 
evaluation". IEEE Transactions on Learning Technologies, vol. 2, no. 3, pp. 203-215, 2009.

[43] A. Mabbott \& S. Bull. "Student Preferences for Editing, Persuading and Negotiating the Open Learner Model". In Proceedings of the Eighth International Conference on Intelligent Tutoring Systems. pp. 481-490, 2006.

[44] E. Martin \& P. Paredes. "Using Learning Styles for Dynamic Group Formation in Adaptive Collaborative Hypermedia Systems". In Workshops in connection with $4^{\text {th }}$ International Conference on Web Engineering. pp. 188-197, 2004.

[45] R. Mayer \& M. Wittrock. Problem-solving transfer. Handbook of Educational Psychology, D. Berliner and R. Calfee, (eds.), Mahwah, NJ: Erlbaum, pp. 47-62, 2006.

[46] E. Melis, E. Andrès, J. Büdenbender, A. Frishauf, G. Goguadse, P. Libbrecht, M. Pollet, \& C. Ullrich. "ActiveMath: A web-based learning environment". International Journal on Artificial Intelligence in Education, vol. 12, no. 4, pp. 385-407, 2001.

[47] A. Moore, T.J. Brailsford, \& C.D. Stewart. "Personally tailored teaching in WHURLE using conditional transclusion". In Proceedings of the Twelfth ACM Conference on Hypertext and Hypermedia, 2001.

[48] Y. E. A. Mustafa \& S.M. Sharif. "An approach to Adaptive E-Learning Hypermedia System based on Learning Styles (AEHS-LS): Implementation and evaluation". International Journal of Library and Information Science, vol. 3, no. 1, pp. 15-28, 2011.

[49] J. Nakic, A. Granic, \& V. Glavinic. "Anatomy of Learner Models in Adaptive Learning Systems: A Systematic Literature Review of Individual Differences from 2001 to 2013". Journal of Educational Computing Research, vol. 51, no. 4, pp. 459-489, 2015.

[50] W. Nejdl \& M. Wolpers. "KBS Hyperbook - A DataDriven Information System on the Web". In Proceedings of the Eighth International Conference on World Wide Web, 1999.

[51] J. Nielsen. Designing Web Usability: The practice of simplicity, USA: New Rider Publishing, 2000.

[52] A. Papadimitriou \& G. Gyftodimos. "Use of Kolb's Learning Cycle through an Adaptive Educational Hypermedia System for a Constructivist Approach of Electromagnetism". In Proc. of WSEAS/IASME International Conference on Engineering Education, pp. 226-231, 2007.

[53] A. Papadimitriou, M. Grigoriadou \& G. Gyftodimos. "A Web-Based Learner-Controlled Adaptive Group Formation Technique". International Journal of eCollaboration, vol. 10, no. 1, pp. 14-34, 2014.

[54] K.A. Papanikolaou, \& M. Grigoriadou. "Combining adaptive hypermedia with project and case based learning". International Journal of Educational Multimedia and Hypermedia, vol. 18, no.2, 2009.

[55] K.A. Papanikolaou, M. Grigoriadou, H. Kornilakis, \& G. Magoulas. "Personalizing the Interaction in a Web-based Educational Hypermedia System: the case of INSPIRE". User-Modelling and User-Adapted Interaction, vol. 13, no. 3, pp. 213-267, 2003.

[56] E. Popescu, P. Trigano, \& C. Badica. "Towards a Unified Learning Style Model in Adaptive Educational Systems". In Proceedings of the ICALT Conference. pp. 804-808, 2007.

[57] E. Popescu. "Adaptation provisioning with respect to learning styles in a Web-based educational system: an experimental study". Journal of computer assisted learning, vol. 26, no. 4, 2010.

[58] C. Portugal, R. M. de Souza Couto. "Educational Support for Hypermedia Design". International Journal of Modern Education and Computer Science (IJMECS), vol.4, no.6, pp. 9-16, 2012.

[59] C.M. Reigeluth, \& F.S. Stein. The Elaboration Theory of Instruction. Instructional design theories and models: An overview of their current status. In C.M. Reigeluth, (Ed.), Hillsdale, N.J.: Lawrence Elrbaum Associates, 1983.

[60] K. Scheiter, P. Gerjets, B. Vollmann, \& R. Catrambone. "The impact of learner characteristics on information utilization strategies, cognitive load experienced, and performance in hypermedia learning". Learning and Instruction, vol. 19, no. 5, pp. 387-401, 2009.

[61] S. Schiaffino, P. Garcia, \& A. Amandi. "eTeacher: providing personalized assistance to e-learning students". Computers \& Education, vol. 51, pp. 1744-1754, 2008.

[62] V.J. Shute \& D. Zapata-Rivera. "Adaptive technologies". In J. M. Spector, D. Merrill, J. van Merriënboer, \& M. Driscoll (Eds.), Handbook of Research on Educational Communications and Technology (3rd Edition) New York: Lawrence Erlbaum Associates, Taylor \& Francis Group, pp. 277-294, 2008.

[63] E. Soloway, S.L. Jackson, J. Klein, C. Quintana, J. Reed, J. Spitulnik, S.J. Stratford, S. Studer, J. Eng, and N. Scala. "Learning theory in practice: Case studies of learnercentered design". In Proc. of the Human Factors in Computing Systems: CHI '96, 1996.

[64] M. Specht \& A. Kobsa. "Interaction of Domain Expertise and Interface Design in Adaptive Educational Hypermedia". In Proceedings of the Second Workshop on Adaptive Systems and User Modelling on the World Wide Web. pp. 89-93, 1999.

[65] M. Specht \& R. Klemke. "ALE - Adaptive Learning Environment". In Proceedings of the World Conference on WWW and Internet, 2001.

[66] M. Specht, G. Weber, S. Heitmeyer, \& V. Schöch. "AST: Adaptive WWW Courseware for Statistics". In Proceedings of the Workshop on Adaptive Systems and User Modelling on the World Wide Web at Sixth International Conference on User Modelling (UM '97), pp. 91-95, 1997.

[67] M. Specht \& R. Oppermann. "ACE-Adaptive Courseware Environment". New Review of Hypermedia and Multimedia, vol. 4, pp. 141-161, 1998.

[68] M.K. Stern \& B.P. Woolf. "Adaptive Content in an Online Lecture System". Adaptive Hypermedia and Adaptive Web-based Systems, (LNCS), 1892, 227-238, 2000.

[69] H. Surjono \& J. Maltby. "Adaptive educational hypermedia based on multiple student characteristics". In Proceedings of the $2^{\text {nd }}$ international conference on webbased learning, pp. 442-449, 2003.

[70] M. D. Svinicki \& N. M. Dixon. "The Kolb's Model Modified for Classroom Activities". Journal of College Teaching, 35(4), 141-146, 1987.

[71] M. A. Tadlaoui, S. Aammou, M. Khaldi, R. N. Carvalho, "Learner Modeling in Adaptive Educational Systems: A Comparative Study". International Journal of Modern Education and Computer Science (IJMECS), Vol.8, No.3, pp.1-10, 2016. DOI: 10.5815/ijmecs.2016.03.01

[72] E. Triantafillou, A. Pomportsis, \& E. Georgiadou. "AESCS: Adaptive Educational System based on Cognitive Styles". In Proceedings of the Workshop on Adaptive Hypermedia (AH2002). pp. 10-20, 2002.

[73] N. Tsianos, P. Germanakos, \& C. Mourlas. "Assessing the Importance of Cognitive Learning Styles over Performance in Multimedia Educational Environments". In Proceedings of the $2^{\text {nd }}$ International Conference on 
Interdisciplinarity in Education (ICIE2006), pp. 123-130, 2006.

[74] L.V. Velsen, T. V. Der Geest, R. Klaassen, \& M. Steehouder. "User-centred evaluation of adaptive and adaptable systems: a literature review". The Knowledge Engineering Review, vol. 23, pp. 261-281, 2008.

[75] G. Weber, H.-C. Kuhl, \& S. Weibelzahl. "Developing adaptive internet based Courses with the authoring system NetCoach". In: P. D. Bra, P. Brusilovsky and A. Kobsa (eds.) In Proceedings of Third workshop on Adaptive Hypertext and Hypermedia, pp. 35-48, 2001.

[76] C. Wolf. "iWeaver: Towards an Interactive Web-Based Adaptive Learning Environment to Address Individual Learning Styles", European Journal of Open Distance Learning, 2002.

\section{Authors' Profiles}

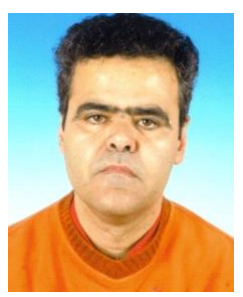

Alexandros Papadimitriou received the diploma from the Department of Electrical and Computer Engineering, National Technical University of Athens (NTUA), in 1992, the MSc degree from the Department of Mechanical Engineering, NTUA, in 2004, and the PhD degree in computer science from the Department of Informatics and Telecommunications, University of Athens, in 2010.

$\mathrm{He}$ is now a Visited Lecturer in the Higher School of Pedagogical and Technological Education in Athens, Greece, and a School Advisor in the Greek ministry of education. His current research interests include the areas of adaptive educational hypermedia systems, adaptive group formation and peer help, interactive problem solving support, meta-adaptation techniques, and didactics of science and technology.

Dr. Papadimitriou was the recipient of the Outstanding Paper Award of the ED-MEDIA 2008 Conference on Educational Multimedia, Hypermedia, and Telecommunications; he was also the recipient of the Best Poster Paper Award of the IEEE ICALT 2009 International Conference on Advanced Learning Technologies.

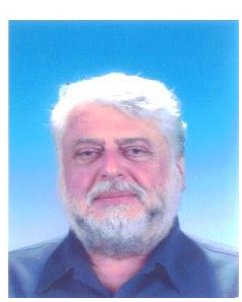

Georgios Gyftodimos received a degree in mathematics and a $\mathrm{PhD}$ degree in informatics. He is currently a retired Associate Professor in the Department of Philosophy and History of Science, University of Athens, Greece where he participates in the Interdisciplinary Postgraduate Program on Cognitive Science and teaches courses in AI, evolutionary programming, and simulation. His research interests lie in the domains of knowledge representation and modelling for cognitive purposes. Associate Professor Gyftodimos is an IEEE fellow.

How to cite this paper: Alexandros Papadimitriou, Georgios Gyftodimos, "The Role of Learner Characteristics in the Adaptive Educational Hypermedia Systems: The Case of the MATHEMA", International Journal of Modern Education and Computer Science(IJMECS), Vol.9, No.10, pp. 55-68, 2017.DOI: 10.5815/ijmecs.2017.10.07 